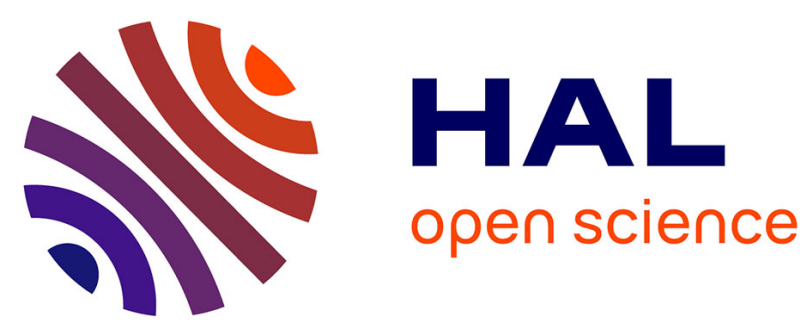

\title{
Photonic crystals versus diffraction gratings in Smith-Purcell radiation
}

\author{
Syuichi Yamaguti, Jun-Ichi Inoue, Olivier Haeberlé, Kazuo Ohtaka
}

\section{To cite this version:}

Syuichi Yamaguti, Jun-Ichi Inoue, Olivier Haeberlé, Kazuo Ohtaka. Photonic crystals versus diffraction gratings in Smith-Purcell radiation. Physical Review B: Condensed Matter and Materials Physics (1998-2015), 2002, 66, pp.N/A. 10.1103/PhysRevB.66.195202 . hal-00956278

\section{HAL Id: hal-00956278 https://hal.science/hal-00956278}

Submitted on 6 Mar 2014

HAL is a multi-disciplinary open access archive for the deposit and dissemination of scientific research documents, whether they are published or not. The documents may come from teaching and research institutions in France or abroad, or from public or private research centers.
L'archive ouverte pluridisciplinaire HAL, est destinée au dépôt et à la diffusion de documents scientifiques de niveau recherche, publiés ou non, émanant des établissements d'enseignement et de recherche français ou étrangers, des laboratoires publics ou privés. 


\title{
Photonic crystals versus diffraction gratings in Smith-Purcell radiation
}

\author{
Syuichi Yamaguti, ${ }^{1}$ Jun-ichi Inoue, ${ }^{2}$ Olivier Haeberlé, ${ }^{3}$ and Kazuo Ohtaka ${ }^{2}$ \\ ${ }^{1}$ Department of Applied Physics, Faculty for Engineering, Chiba University, 1-33 Yayoi-cho, Inage-ku, Chiba 263-8522, Japan \\ ${ }^{2}$ Center for Frontier Science, Chiba University, 1-33 Yayoi-cho, Inage-ku, Chiba 263-8522, Japan \\ ${ }^{3}$ Group LabEl Laboratoire MIPS Université de Haute-Alsace IUT de Mulhouse, 61 rue Albert Camus, 68093 Mulhouse Cedex, France
}

(Received 9 July 2002; revised manuscript received 3 September 2002; published 7 November 2002)

\begin{abstract}
The emission cross section of Smith-Purcell radiation is calculated for a charged particle running near the surface of a photonic crystal. The cross section of a photonic crystal of arrayed dielectric spheres and that of a diffraction grating are compared. It is shown that well-defined photonic bands of the photonic crystal bring about a spectrum full of resonant structures of enhanced intensity, whose peak heights are an order of magnitude larger than the emission intensity of the diffraction grating. The results of calculations thus show a possible advantage of photonic crystals over diffraction gratings as a converter of the evanescent field from a running charge into propagating waves.
\end{abstract}

DOI: 10.1103/PhysRevB.66.195202

PACS number(s): 42.70.Qs, 41.20.Jb

\section{INTRODUCTION}

Smith-Purcell radiation (SPR) is a mechanism of photon emission from a charge running near a periodic array of scatterers of electromagnetic waves. The evanescent field emitted by the running charge, present only close to its trajectory and hence unable to be observed at a distant observation point, acquires an umklapp momentum shift due to scattering and is eventually converted into an observable plane wave. Since the pioneering discovery of this light conversion process by Smith and Purcell, ${ }^{1}$ diffraction gratings have been conventionally employed to make use of the onedimensional (1D) umklapp scattering by surface corrugations. Because of the easiness of the fabrication, the study using the 1D periodicity of diffraction gratings has been actively pursued and the possible applications in the new fields of laser acceleration ${ }^{2,3}$ and in free electron lasing ${ }^{4,5}$ have been studied both theoretically and experimentally.

The well definedness of photonic bands of photonic crystals ${ }^{6,7}$ naturally leads to the expectation that production of photons of higher efficiency might be achieved if a photonic crystal is used instead of a diffraction grating. The band structure of a photonic crystal introduces frequency ranges of very low density of states (DOS) of photons, if not completely zero as realized in the presence of a full band gap. The low DOS frequency range centering on a photonic band gap is sandwiched by a pair of photonic bands of very small group velocity, sometimes as small as one hundredth of the light velocity $c$ in vacuo. A lowered group velocity of light is equivalent to an enhanced photonic DOS. Therefore, the SPR spectrum of a photonic crystal may exhibit frequency ranges of enhanced intensity at the positions of photonic bands to compensate the suppression of intensity at the band gap regions.

In this sense, the efficiency of a photonic crystal in the Smith-Purcell (SP) mechanism is quite an intriguing target in the search for the future applications of photonic crystals. In fact, calculations given by Ohtaka and Yamaguti for a photonic crystal of slab geometry show that resonant excitation of a photonic band by the evanescent light from an electron gives rise to conspicuous resonant peaks in the SPR spectrum. ${ }^{8}$ They found that the resonant enhancement grow progressively as the slab becomes thicker.

In the case of diffraction gratings ${ }^{9-14}$ and $1 \mathrm{D}$ periodic scatterers, ${ }^{15}$ too, the SPR spectrum has a nontrivial frequency dependence, as observed both experimentally and theoretically. The spectrum is often characterized by the appearance of frequency ranges of enhanced intensity, in connection with the opening of a diffraction channel as the photon energy increases. This is a kind of Wood anomaly, the name given to any singular behavior in grating optics that takes place associated with the opening of a diffraction channel. ${ }^{16-20}$ As compared with the resonances in photonic crystal, however, such frequency ranges of enhancement would presumably be much broader and the peak heights of the grating SPR spectra would accordingly be so much smaller. This conjecture stems from the fact that the normal modes of a photon, if any, of a grating system cannot be established so well as the photonic bands of photonic crystal. In this regard, it should be noted that the sharp resonances in many optical responses of photonic crystals result from the remarkable confinement effect of electromagnetic energies due either to the strong Bragg reflection or from photon localization as in the case of Mie resonance.

The purpose of this paper is to examine the efficiency of photonic crystals in SPR compared with that of diffraction gratings. One of our targets is to compare the spectral features of 2D light scattering of photonic crystals with that of diffraction gratings. By considering a photonic crystal of finite thickness, we shall show that it gives an SPR spectrum full of information, so much so that we can even observe the dispersion curves of the photonic bands by following the peak positions in the SPR spectrum using the scanning direction of observation and/or changing beam velocity. We shall also show that the SPR spectrum from a photonic crystal presents a selection rule related to the photonic band structures.

The second target is to compare the absolute magnitudes of photon production. For this purpose, we shall calculate the absolute magnitudes of SPR from a photonic crystal as a function of the direction of observation. The conclusion to be drawn for the efficiency of photon yield is very positive for 


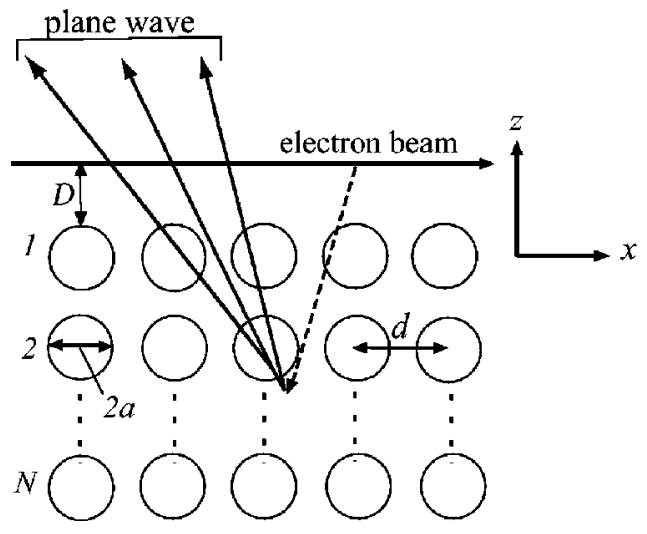

FIG. 1. Electron beam and a slab photonic crystal of arrayed spheres. The electron is running in the $x$ direction with distance $D$ from the top of the first layer spheres. Dielectric spheres, each of radius $a$ are arrayed in a simple cubic array of lattice constant $d . N$ is the number of stacking layers. The evanescent wave shown by the dashed line is converted into plane waves, shown by the solid lines, by the umklapp light scattering.

the photonic crystal over a perfectly conducting grating both quantitatively and qualitatively.

These conclusions are drawn for the SPR spectra from a slab photonic crystal of arrayed spheres. The system of arrayed spheres is chosen because it is a prototype photonic crystal that might cover any characteristic optical phenomena realizable in photonic crystals. The conclusions of this paper may thus be taken as the characteristic features of photonic crystals in general and may be applicable to any realistic photonic crystals of sophisticated fabrication except, of course, for numerical details, which may differ from one photonic crystal to another.

In Sec. II, our theoretical treatment in SPR spectrum will be given. In Sec. III, we give some of the numerical results of the SPR spectra in connection with the band structure of the photonic crystal. In Sec. IV, the formula for calculating the absolute magnitudes of SPR spectra is given and a comparison is made between a photonic crystal and a perfectly conducting grating. The conclusions are given in Sec. V.

\section{SPR MECHANISM OF PHOTONIC CRYSTAL}

We consider an electron of charge $-e(e>0)$ running with velocity $v$, parallel to the surface of a photonic crystal of finite thickness. As shown in Fig. 1, we examine a photonic crystal made of dielectric spheres arrayed in a simple cubic lattice of lattice constant $d$. We place the centers of the spheres of the first layer on the $x-y$ plane $(z=0)$, with the $x$ axis taken in the $(1,0)$ direction of the square lattice and put the whole system composed of $N$ stacking layers in the region $z<0$. The lateral extension in the $x$ and $y$ directions of the photonic crystal is assumed to be infinite. Let the electron be running in the $x$ direction with distance $D+a$ above the $x-y$ plane, where $a$ is the radius of spheres and $D$ is the distance between the electron trajectory and the top of the first-layer spheres.

Since we are interested in the frequency region of photons, which are influenced substantially by the photonic crys- tal, the frequency $\omega$ of the emitted photon can be assumed to be in the visible or longer wavelength range. With $\hbar \omega$ much smaller than the beam energy, which we assume to be in the hundreds of kilovolts or higher in the relativistic range, the recoil of the electron due to the photon emission may be neglected. The emission of light is then treated simply by solving Maxwell equations with the beam current treated as a source term, an assumption usually employed in the treatment of SPR. ${ }^{9-16}$

With the origin of time $t$ fixed at the instant at which the electron passes the point $x=0$, the current of an electron with velocity $v$ is expressed by

$$
j(\mathbf{r}, t)=-e \hat{x} v \delta(x-v t) \delta(y) \delta(z-D-a),
$$

where $\hat{x}$, the unit vector in the $x$ direction, specifies the current direction. The Fourier transform of $\boldsymbol{j}(\mathbf{r}, t)$ with respect to $t, \boldsymbol{j}(\mathbf{r}, \omega)$, works as a source term in Maxwell equations for the emission of a light of frequency $\omega$. We obtain

$$
j(\mathbf{r}, \omega)=-e \hat{x} e^{i k_{x} x} \delta(y) \delta(z-D-a),
$$

with a key relation

$$
k_{x}=\frac{\omega}{v} .
$$

Since the source term $\boldsymbol{j}(\mathbf{r}, \omega)$ exhibits $e^{i k_{x} x}$ dependence, the wave emitted directly by the electron will be characterized by the same dependence. The vector potential of the emitted wave is obtained in the Lorentz gauge by solving

$$
\left(\Delta+\frac{\omega^{2}}{c^{2}}\right) A(\mathbf{r}, \omega)=-\mu_{0} j(\mathbf{r}, \omega)
$$

With Eq. (2), we find

$$
\begin{aligned}
\boldsymbol{A}(\mathbf{r}, \omega)= & \mu_{0} e \hat{x} \int \frac{d q_{y} d q_{z}}{(2 \pi)^{2}} \\
& \times \frac{\exp \left\{i\left[k_{x} x+q_{y} y+q_{z}(z-D-a)\right]\right\}}{\omega^{2} / c^{2}-\left(k_{x}^{2}+q_{y}^{2}+q_{z}^{2}\right)} .
\end{aligned}
$$

Note that the $x$ dependence is described by the factor $e^{i k_{x} x}$, as stated above. The integral over $q_{z}$ of this equation is given by the residue of the integrand. With the Lorentz condition, the electric field is then obtained as

$$
\boldsymbol{E}(\mathbf{r}, \omega)=i \omega\left(\boldsymbol{A}(\mathbf{r}, \omega)+\frac{c^{2}}{\omega^{2}} \nabla \boldsymbol{\nabla} \cdot \boldsymbol{A}(\mathbf{r}, \omega)\right),
$$

the second term being the contribution from the scalar potential. Substituting Eq. (5) into Eq. (6), we find

$$
\boldsymbol{E}(\mathbf{r}, \omega)=\frac{e}{2 c^{2} \varepsilon_{0}} \omega \int \frac{d q_{y}}{2 \pi}\left(-1+\frac{1}{\beta^{2}}, \frac{q_{y}}{\kappa \beta}, \frac{\Gamma}{\kappa \beta}\right) \frac{e^{i \mathbf{k}_{i} \cdot \mathbf{r}}}{\Gamma},
$$

where $\kappa=\omega / c, \beta=v / c$ and $\varepsilon_{0}$ is the dielectric constant of vacuum. Here, 


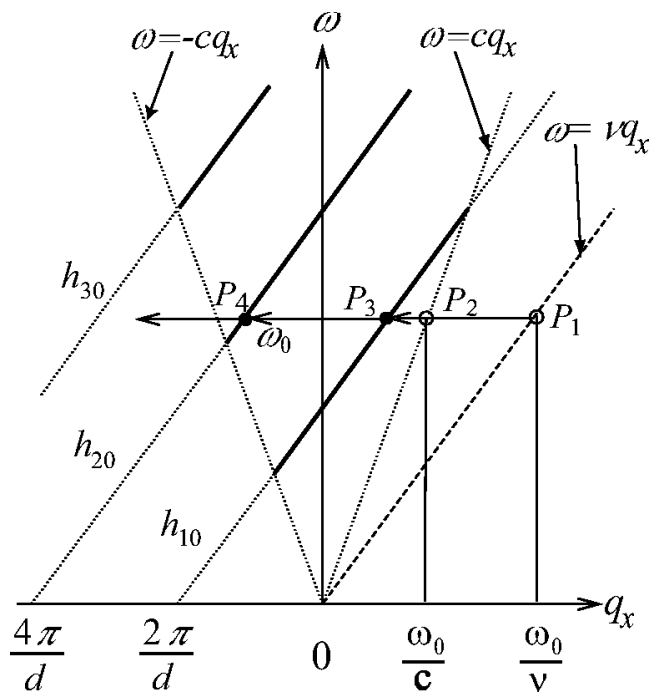

FIG. 2. Light conversion using a umklapp momentum shift. The dashed lines show the dispersion relation $\omega=v q_{x}$ of the evanescent wave (called the $v$ line in the text) and those umklapp shifted in parallel to it ( $h_{10}$ and $h_{20}$ lines). The lattice constant of the reciprocal lattice is $2 \pi / d$. The shift from point $P_{1}$ to $P_{3}$ or $P_{1}$ to $P_{4}$ corresponds to the conversion process of the evanescent wave of frequency $\omega_{0}$. The light lines $\omega= \pm c q_{x}$ define the light cone. The part of a shifted $v$ line bounded by them (shown by the solid line) defines the SPR band. The $q_{x}$ coordinates of the two points $P_{1}$ and $P_{2}, \omega_{0} / v$ and $\omega_{0} / c$, respectively, determine the damping constant $|\Gamma|$ through Eq. (9).

$$
\mathbf{k}_{i}=\left(k_{x}, q_{y}, \Gamma\right)
$$

is the wave vector of the emitted photon with

$$
\Gamma=\sqrt{\kappa^{2}-k_{x}^{2}-q_{y}^{2}}=\sqrt{\left(\frac{\omega}{c}\right)^{2}-\left(\frac{\omega}{v}\right)^{2}-q_{y}^{2}}
$$

being its $z$ component. Note that $q_{y}$ of the wave vector $\mathbf{k}_{i}$ is arbitrary and that the integral over $q_{y}$ remains in Eq. (7) for $\boldsymbol{E}(\mathbf{r}, \omega)$. Since

$$
v<c
$$

in Eq. (9), $\Gamma$ is a pure imaginary number, implying that the direct light from the charge, Eq. (7), is evanescent in the $z$ direction. We illustrate this situation in Fig. 2, taking the case of $q_{y}=0$, for simplicity. The two dotted lines are drawn in the $q_{x}-\omega$ plane for the dispersion relations $\omega=c q_{x}$ and $\omega$ $=v q_{x}$. They will be referred to as $c$ line and $v$ line, respectively. The two intersections, $P_{1}$ and $P_{2}$ in Fig. 2, determine the magnitude of $\Gamma$, as we see from Eq. (9).

We now treat the evanescent wave given by Eq. (7) as an incident wave of wave vector $\mathbf{k}_{i}$ to the photonic crystal. It is multiply scattered and goes out of the crystal after the scattering process. Since translational invariance is lacking in a photonic crystal of finite thickness, the reciprocal lattice vectors in question are 2D ones. Each of the reciprocal lattice vectors determines one SPR band.

In the upper side of the photonic crystal, reflected wave is observed with the momentum shift due to a $2 \mathrm{D}$ reciprocal lattice vector

$$
\left(\Delta q_{x}, \Delta q_{y}\right)=\frac{2 \pi}{d}\left(n_{x}, n_{y}\right)
$$

with integers $n_{x}$ and $n_{y}$. The wave vector of the scattered light is then given by

$$
\mathbf{k}_{s}\left(n_{x}, n_{y}\right)=\left(k_{x}-n_{x} \frac{2 \pi}{d}, q_{y}-n_{y} \frac{2 \pi}{d}, \Gamma_{n_{x} n_{y}}\right),
$$

with

$$
\Gamma_{n_{x} n_{y}}=\sqrt{\kappa^{2}-\left(k_{x}-n_{x} \frac{2 \pi}{d}\right)^{2}-\left(q_{y}-n_{y} \frac{2 \pi}{d}\right)^{2}}
$$

by energy conservation. The $\left(n_{x}, n_{y}\right)$ waves with a real $\Gamma_{n_{x} n_{y}}$, i.e., the waves with $\mathbf{k}_{s}\left(n_{x}, n_{y}\right)$ lying inside the light cone of Fig. 2, can propagate as a plane wave and are detectable at a distant observation point. Figure 2 shows the construction of these propagating waves for the case of $n_{y}$ $=0$. We have a series of $v$ lines shifted horizontally by the quantity $-\left(2 \pi n_{x} / d\right)$. In the frequency region within the light cone, i.e., along a shifted $v$ line bounded by the two $c$ lines $\omega= \pm c q_{x}$, we have one SPR emission band. Thus, the entire spectrum is composed of $h_{10}, h_{20}, \ldots$, emission bands, where the subscript 10, for example, specifies the momentum shift $\left(n_{x}, n_{y}\right)=(1,0)$. The angle of the emitted photon relative to the $z$ axis is determined by the direction of $\mathbf{k}_{s}$. With a change in $\omega$ and hence in $k_{x}$ of the initial evanescent light, $\mathbf{k}_{s}$ moves on the shifted $v$ line.

For the treatment of the light scattering of a slab of stacking layers $N$ equal to $2^{n}$, we employ the layer-doubling method. $^{21-23}$ For details of the process of doubling the number of layers, the readers are referred to Ref. 24 Note that the assumption of finite thickness of our system is important because actual experiments are carried out only for such a system and the SPR spectrum depends critically upon the slab thickness. In this way, we can obtain the amplitude transmission and reflection coefficients of the incident evanescent wave of $\mathbf{k}_{i}$. Since the light reflected back is our concern, the amplitude reflection coefficient $\boldsymbol{R}\left(\mathbf{k}_{s} ; \mathbf{k}_{i}\right)$ of an $N$-layer photonic crystal must be obtained. The quantity $\boldsymbol{R}\left(\mathbf{k}_{s} ; \mathbf{k}_{i}\right)$ is a $3 \times 3$ tensor specified by three cartesian components of incident light and scattered light; $\boldsymbol{R}\left(\mathbf{k}_{s} ; \mathbf{k}_{i}\right)_{x y}$, for example, is defined to be the amplitude of the $x$ component of the reflected light of $\mathbf{k}_{s}$ obtained from the $y$-polarized incident light of $\mathbf{k}_{i}$ of unit amplitude. Recovering the $q_{y}$ integral of Eq. (7), we obtain the reflected wave after the scattering in the following form:

$$
\boldsymbol{E}_{s}(\mathbf{r}, \omega)=\frac{e}{4 \pi \varepsilon_{0} c} \sum_{n_{x} n_{y}} \int d q_{y} \exp \left(i \mathbf{k}_{s} \cdot \mathbf{r}\right) \boldsymbol{E}_{1}^{\left(n_{x} n_{y}\right)}\left(k_{x}, q_{y}, \Gamma\right),
$$

where

$$
\boldsymbol{E}_{1}^{\left(n_{x} n_{y}\right)}\left(k_{x}, q_{y}, \Gamma\right)=\boldsymbol{R}\left(\mathbf{k}_{s} ; \mathbf{k}_{i}\right) \boldsymbol{E}_{0}\left(k_{x}, q_{y}, \Gamma\right) .
$$

Here, $\boldsymbol{E}_{0}$ is the amplitude of the incident wave on the first layer with the irrelevant prefactor of Eq. (7) removed: 


$$
\boldsymbol{E}_{0}\left(k_{x}, q_{y}, \Gamma\right)=\omega\left(-1+\frac{1}{\beta^{2}}, \frac{q_{y}}{\kappa \beta}, \frac{\Gamma}{\kappa \beta}\right) \frac{\mathrm{e}^{-|\Gamma|(D+a)}}{c \Gamma} .
$$

Since $c \Gamma$ has the dimension of $\omega$ in this equation, $\boldsymbol{E}_{0}$ is a dimensionless quantity and takes account of the decay of the evanescent light through the exponential factor. We define SPR intensity in the following form:

$$
I\left(n_{x}, n_{y}\right)_{q_{y}}=\left|\boldsymbol{E}_{1}^{\left(n_{x} n_{y}\right)}\left(k_{x}, q_{y}, \Gamma\right)\right|^{2} .
$$

A set $\left(n_{x}, n_{y}\right)$ defines one SPR band. When $q_{y}$ and $\omega$ are both fixed, a sharp spot is obtained in the $\left(n_{x}, n_{y}\right)$ band because $\mathbf{k}_{s}$ is uniquely determined by Eq. (12). The spot that has the highest intensity of all will be that of $\left(n_{x}, n_{y}\right)$ $=(1,0)$, the band with the shortest umklapp shift, which appears along the $h_{10}$ line of Fig. 2. If we sweep $\omega$ in the $(1,0)$ band i.e., in the case $n_{y}=0$, the spot moves because $k_{x}$ of $\mathbf{k}_{i}$, and hence $k_{x}-2 \pi / d$ of $\mathbf{k}_{s}$, changes with $\omega$, thus producing the $h_{10}$ SPR band along the $h_{10}$ line. If we sweep $q_{y}$ in addition, the direction of $\mathbf{k}_{s}$ varies in the $y$ direction, too. If, in particular, we restrict the direction of observation to within the $x-z$ plane, the observed intensity is given by the contribution from $q_{y}=0$. To describe the SPR intensity of this situation, we consider

$$
I(1,0)_{0}=\left|\boldsymbol{E}_{1}^{(10)}\left(k_{x}, 0, \Gamma\right)\right|^{2}
$$

by putting $n_{y}=q_{y}=0$ in Eq. (17) and call this quantity simply the SPR intensity of the $h_{10}$ band. The emitted light of $\omega$ propagates in the $x-z$ plane of an angle $\theta$ to the beam direction ( $x$ axis), given by

$$
\cos \theta=\frac{k_{x}-2 \pi / d}{\omega / c} .
$$

Thus, in the direction perpendicular to the electron beam $(\theta=\pi / 2)$, a photon of frequency $\omega=2 \pi c / d$ (i.e., $k_{x}$ $=2 \pi / d)$ is observed. In the same way, the intensity $I\left(n_{x}, 0\right)_{0}$ defined by

$$
I\left(n_{x}, 0\right)_{0}=\left|\boldsymbol{E}_{1}^{\left(n_{x} 0\right)}\left(k_{x}, 0, \Gamma\right)\right|^{2}
$$

with $n_{x}=2,3, \ldots$, produces bands along the $h_{20}, h_{30}, \ldots$, lines in Fig. 2, which are still observed in the $x-z$ plane.

The contribution to the emission in the $x-z$ plane comes not only from $\boldsymbol{E}_{1}^{\left(n_{x} 0\right)}\left(k_{x}, 0, \Gamma\right)$ but also from

$$
\boldsymbol{E}_{1}^{\left(n_{x} n_{y}\right)}\left(k_{x}, q_{y}, \Gamma\right)
$$

with $q_{y}=n_{y}(2 \pi / d)$, because $\left(\mathbf{k}_{s}\right)_{y}$, the $y$ component of the scattered wave vector, is still

$$
\left(\mathbf{k}_{s}\right)_{y}=q_{y}-n_{y} \frac{2 \pi}{d}=0 .
$$

Accordingly, we define the intensity

$$
I\left(n_{x}, n_{y}\right)_{n_{y}}=\left|\boldsymbol{E}_{1}^{\left(n_{x} n_{y}\right)}\left(k_{x}, n_{y} \frac{2 \pi}{d}, \Gamma\right)\right|^{2}
$$

for the emission in the $x-z$ plane [for simplicity, we have put the suffix $q_{y}$ of this $I\left(n_{x}, n_{y}\right)_{q_{y}}$ in units of $\left.2 \pi / d\right]$.

If $I(1,1)_{1}$, say, has a comparable magnitude to $I(1,0)_{0}$ defined by Eq. (20), we should consider the sum

$$
\boldsymbol{E}_{1}^{(10)}\left(k_{x}, 0, \Gamma\right)+\boldsymbol{E}_{1}^{(11)}\left(k_{x}, \frac{2 \pi}{d}, \Gamma\right)
$$

to take into account the interference between the two fields instead of treating them separately.

The final quantity which concerns us is $I\left(n_{x}, 0\right)_{q_{y}}$ defined by

$$
I\left(n_{x}, 0\right)_{q_{y}}=\left|\boldsymbol{E}_{1}^{\left(n_{x} 0\right)}\left(k_{x}, q_{y}, \Gamma\right)\right|^{2} .
$$

It determines the emisssion intensity in the direction deviated from the $x-z$ plane by the finite value of $\left(\mathbf{k}_{s}\right)_{y}\left(=q_{y}\right)$. If $q_{y}$ gradually increases from zero, it provides us with the intensity change as the angle of observation is tilted in the $y$ direction from the $x-z$ plane. In the next section these three SPR intensities are successively examined.

\section{SPR SPECTRUM AND THE BAND STRUCTURE OF SLAB PHOTONIC CRYSTAL}

Smith-Purcell radiation spectra from photonic crystals are expected to have peculiar properties related to the photonic bands. Before entering into a detailed discussion of the SPR spectra, therefore, some comments on the origin and properties of the band structure of a slab photonic crystal will be helpful.

The origin of the band structure in a system of a finite thickness can be easily understood if we begin with the tightbinding-band picture for a single layer of spheres, i.e., a photonic crystal of $N=1$. The Bloch states thereof are formed by the hopping motion of the whispering gallery modes (WGM's). ${ }^{25}$ A WGM of an individual sphere is specified by the angular momentum index $(l, m)$ and hops to adjacent spheres by making use of the incomplete mode confinement and the resulting overlap of the electric fields. The modes set up within the layer therefore have a dispersion due to lateral motion. In this process, the $(2 l+1)$-fold degeneracy of a WGM with respect to $m$ is lifted, though only partly (see Tables IV and V of Ref. 26 for how the degeneracy is resolved). Even in the slab of $N=1$, therefore, the band structure is rather complicated. For the system of $N=2$, the band structures of each of the two layers, which would be twofold degenerate if they were far apart, become mixed to lift the degeneracy. Roughly speaking, the number of bands of the system of $N=2$ is two times larger than that of the system of $N=1$. In the system of $N=3$, the threefold degeneracy of the single-layer band structure is split. In this way, the density of the bands increases progressively with $N$.

Calculation of the band structure is not at all simple. We did this calculation exactly by constructing the $S$ matrix using the transmission and reflection amplitudes of an $N$-layers system and by plotting the peak positions of the calculated density of states as a function of the lateral wave vector. See the algorithm for obtaining the density of states from an $S$ 

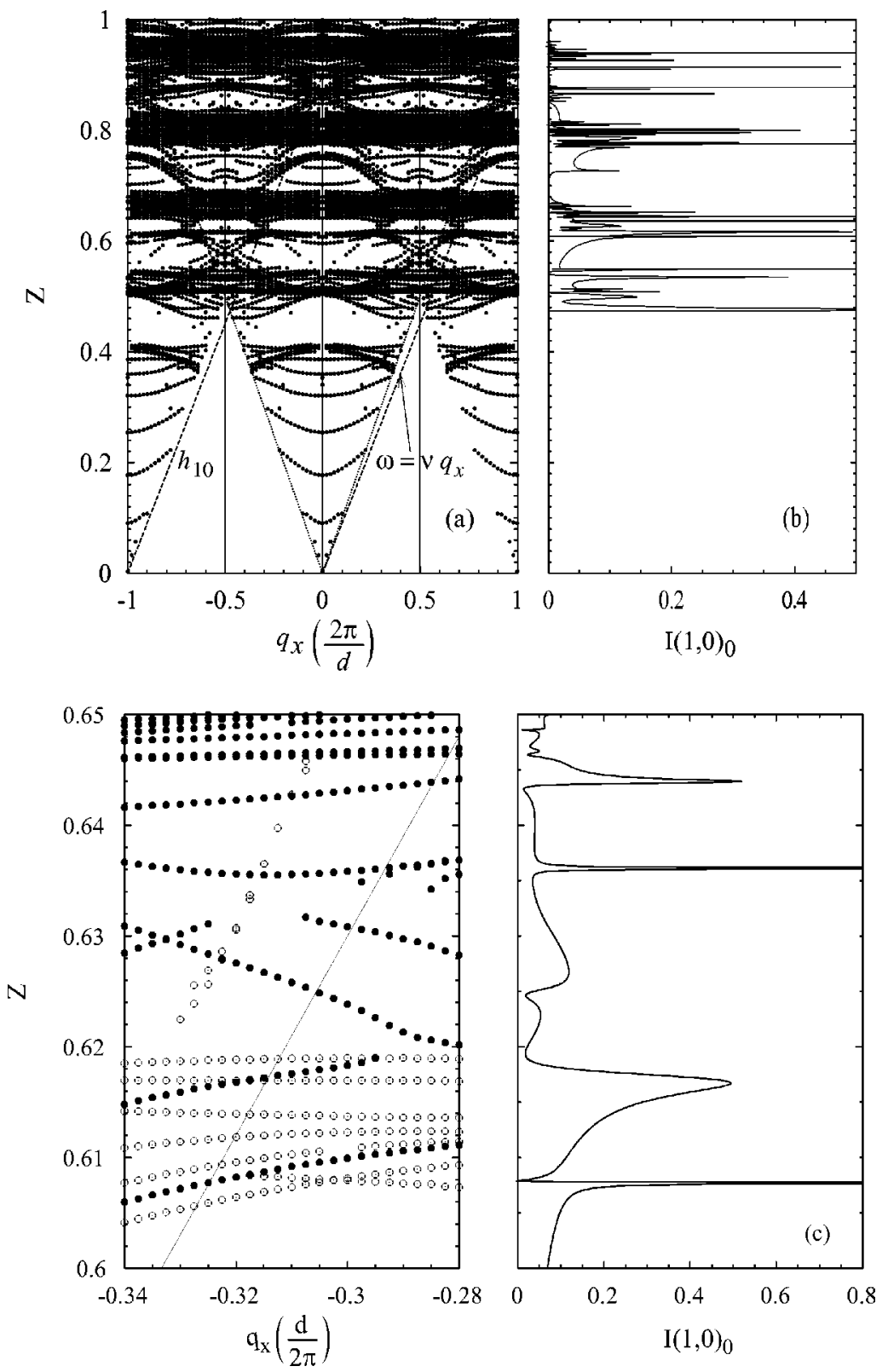

FIG. 3. Band structure and SPR spectrum on the $h_{10}$ line. The panel (a) shows the band structure for $\mathbf{q}=\left(q_{x}, 0\right)$, and (b) shows the plot of $I(1,0)_{0}$ defined by Eq. (18) for $\beta=0.9$. In (a), two $c$ lines which define the light cone are shown by dotted lines together with the $v$ line $\omega=v q_{x}$ and the $v$ line shifted by a 2D reciprocal-lattice vector $\mathbf{h}=(2 \pi / d)(1,0)\left(h_{10}\right.$ line $)$. The panel (c) is a reproduction of (a) and (b) on much larger scales. The filled (open) circles of the band structure show the dispersion relations of the $p$-active ( $s$-active) bands. The straight line is the $h_{10}$ line. In the frequency region where the dispersion curves are sparsely populated, the lifetimes of the bands are generally very short. Sometimes they are too short to identify the peak positions of the density of states. This is the reason for the abrupt appearance or disppearance of some of the data points in the band structure. matrix given in Refs. 27 and 28. The difficulty in the band calculation is partly due to the fact that a band of a slab system generally has a finite lifetime caused by the leakage of the mode energy to the exterior region. This is exactly the same feature as that encountered in the treatment of lifetimebroadened WGM's in Mie scattering of light in an isolated sphere. The use of the transmission and reflection coefficients of the external light in the $S$ matrix successfully incorporates this coupling between the inside and outside of the slab system. Since the leak of the electric field is partly consumed in forming a coherent lateral motion and in lifting the degeneracy among the layers, the lifetime becomes longer in a thicker photonic crystal. In other words, the quality factor of the excited modes becomes larger as $N$ increases.

\section{A. SPR spectra of $I\left(n_{x}, 0\right)_{0}$ given by Eq. (20)}

First we study the emission in the $x-z$ plane and concentrate on $I(1,0)_{0}$ defined by Eq. (18). This quantity determines the $h_{10}$ SPR band in Fig. 2. We choose $a / d=0.42$ and $D$ $=d / 2$. The dielectric constant $\varepsilon$ of the spheres and the ratio $\beta=v / c$ are taken to be $\varepsilon=3.2^{2}$ and $\beta=0.9$, respectively. The value 3.2 of the refractive index is twice as large as that of polystylene spheres in the visible range. ${ }^{29}$ Unless otherwise stated, these parameters are used throughout the present paper. We use the normalized unit of frequency $Z$ defined by $Z=\omega d / 2 \pi c$.

Figure 3 shows $I(1,0)_{0}$ and the corresponding band structure for the case of $N=4$ with frequency $Z$ taken in the vertical axis. It can be seen that the SPR spectrum shown in Fig. 3(b) is characterized by the presence of many resonant peaks and that these peaks can be roughly divided into groups. The appearance of the groups of resonances reflects that of grouped band energies cut by the $h_{10}$ line shown in Fig. 3(a). The grouping of the dispersion curves is understood in terms of the splitting of the degeneracy mentioned above. To show the coincidence between Figs. 3(a) and 3(b) 

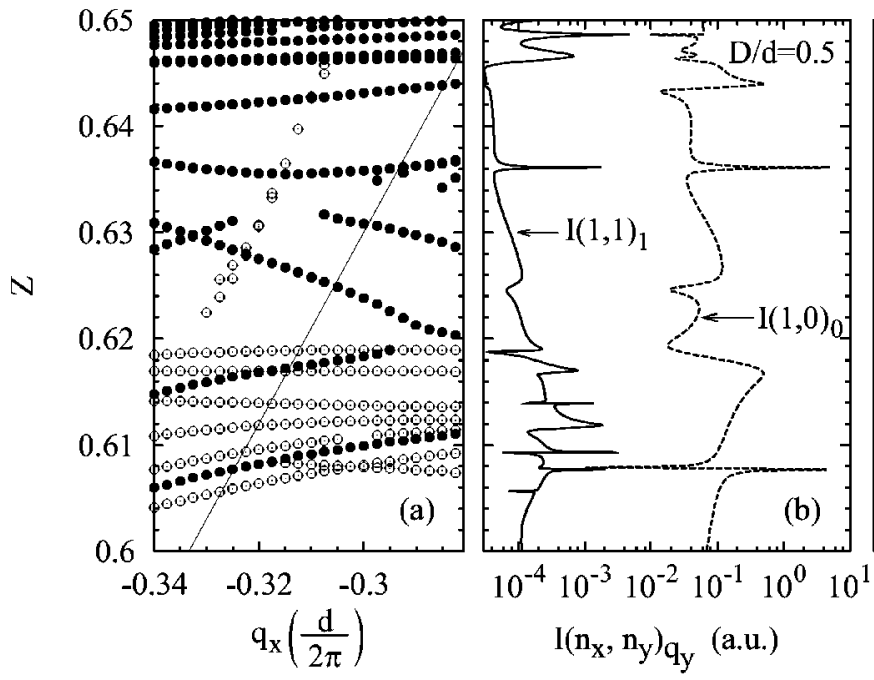

in more detail, we reproduced in (c) a very narrow frequency range of (a) and (b) in larger scales. The solid and open circles in the left panel represent the bands active to $p$ - and $s$-polarized incident lights, respectively. It is obvious that solely the crossings of the $h_{10}$ line with the dispersion curves of the $p$-active bands give rise to the resonant peaks in the right panel. Therefore, it is concluded that the SPR spectrum of $q_{y}=n_{y}=0$ reacts only to the excitation of the $p$-active bands. This selection rule is simply explained by the fact that the evanescent incident light is $p$ polarized when $q_{y}=0$, as seen from the polarization of $\boldsymbol{E}_{0}$ given by Eq. (16); it excites only the $p$-active bands polarized in the $x-z$ plane.

\section{B. SPR spectra of $I\left(n_{x}, n_{y}\right)_{n_{y}}$ given by Eq. (23)}

We next study $I(1,1)_{1}\left(n_{y}=1\right)$, which is a dominant contribution from a finite $q_{y}$ of the initial photon to the $h_{10}$ emission band observed in the $x-z$ plane. The spectrum of $I(1,1)_{1}$ and the related band structure are shown in Figs. 4(a) and 4(b), respectively. Because of the periodicity in the momentum space, the band structure of Fig. 4(a) for $\mathbf{q}$ $=\left(q_{x}, 2 \pi / d\right)$ is identical to that of Fig. 3(c) for $\mathbf{q}=\left(q_{x}, 0\right)$, and the line $h_{11}$ is the same as the $h_{10}$ line of Fig. 3(c). In Fig. 4(b), the solid curve represents $I(1,1)_{1}$, while the dashed curve shows for comparison $I(1,0)_{0}$ examined in Sec. III A. From Figs. 4(a) and 4(b), it can be concluded that not only the $p$ - but also $s$-active bands appear as distinct resonant peaks in $I(1,1)_{1}$ in clear contrast to $I(1,0)_{0}$ examined above. This feature comes from the finiteness of the $y$ component of the incident polarization when $q_{y} \neq 0$; the polarizations of the evanescent wave are neither $p$ nor $s$ and so are the excited photonic bands.

Figure 4 also shows that the intensity $I(1,1)_{1}$ is much smaller than $I(1,0)_{0}$. Through Eq. (9), $|\Gamma|$ becomes larger as $q_{y}$ increases. Therefore, the incident evanescent wave with $q_{y}=2 \pi / d$ decays much faster than does the wave with $q_{y}$ $=0$ considered in $I(1,0)_{0}$. For a larger value of $n_{y}$, $I\left(1, n_{y}\right)_{n_{y}}$ is reduced even further. So that $I(1,1)_{1}$ should be dominant among them. However, it should be noted that in the case of a smaller value of $D$, the exponential damping due to $|\Gamma|$ is reduced. Figure 4(c) shows the results for the

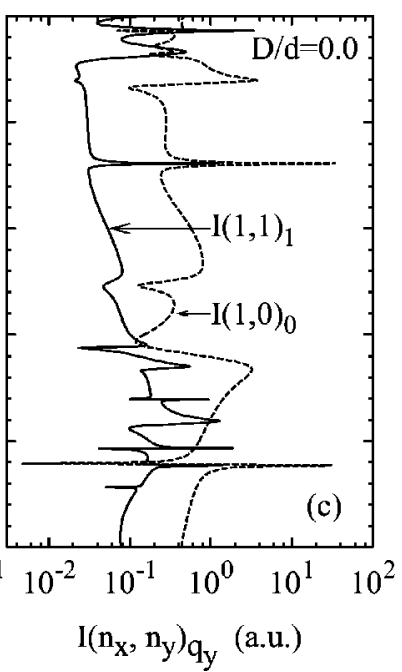

FIG. 4. Band structure for $q_{y}$ $=0$ and SPR intensity $I(1,1)_{1}$. The results for $\beta=0.9$ and $N=4$ are given. The panel (a) is a reproduction of the band structure shown in Fig. 3(c). The panels (b) and (c) plot $I(1,1)_{1}$ defined by Eq. (23), with $D / d=0.5$ for the central figure and with $D / d=0$ for the right figure. $I(1,0)_{0}$ of Fig. 3 is given, for comparison. limiting case $D=0$. We see that at some peak positions due to $I(1,1)_{1}$, the solid-line intensity indeed exceeds the dashed one.

However, $I(1,1)_{1}$ is in general about one-order of magnitude lower than $I(1,0)_{0}$. As a consequence the interference process described by Eq. (24) may in general be neglected. We may hence conclude that the intensity of the $h_{10}$ SP band which would be obtained by the exact sum over $n_{y}$ is practically the same as the contribution solely from $n_{y}=0$. Therefore, the spectra of $I(1,0)_{0}$ shown in Fig. 3 can essentially be taken to be the exact ones of the $h_{10}$ SP band.

The spectra $I\left(n_{x}, 0\right)_{0}$ with $n_{x}=2,3, \ldots$, have been computed and found to be much smaller than $I(1,0)_{0}$. The first reason for the smallness is that they are lying in the frequency regions higher than $n_{x}=1$ (see Fig. 2) and the decay of the incident evanescent wave is so much larger, with the increase of the distance between the points $P_{1}$ and $P_{2}$ in Fig. 2. The second reason is that a larger umklapp momentum shift leads to a smaller conversion rate as in a usual potential scattering of electron. For another system, the comparison of $I(2,0)_{0}$ with $I(1,0)_{0}$, which supports the present conclusion, is found in Ref. 8 .

The next two subsections are related to the experiments which we do hope to be carried out in future. The first is related to how the spectrum changes as the direction of observation of emitted photons is tilted in the $y$ direction. The second concerns the direction of the beam relative to the symmetry axis of photonic crystal. Since these two topics are out of the main stream of this paper, the readers who want in a hurry to make a comparison between photonic crystal and diffraction gratings, can skip the following two subsections and pass directly to Sec. IV.

\section{SPR spectra of $I\left(n_{x}, 0\right)_{q_{y}}$ given by Eq.,(25)}

So far, we have examined the vertical emission of $\left(\mathbf{k}_{s}\right)_{y}$ $=0$. Here, we examine the case of nonzero $\left(\mathbf{k}_{s}\right)_{y}$ concentrating on $I(1,0)_{q_{y}}$ with a finite but small $q_{y}$.

When $q_{y} \neq 0$, the polarization of the incident light is neither $p$ nor $s$ as in Sec. III B. Thus, any band cut by the $h_{10}$ line will leave a distinct trace in the SPR spectra; since $\mathbf{q}$ 


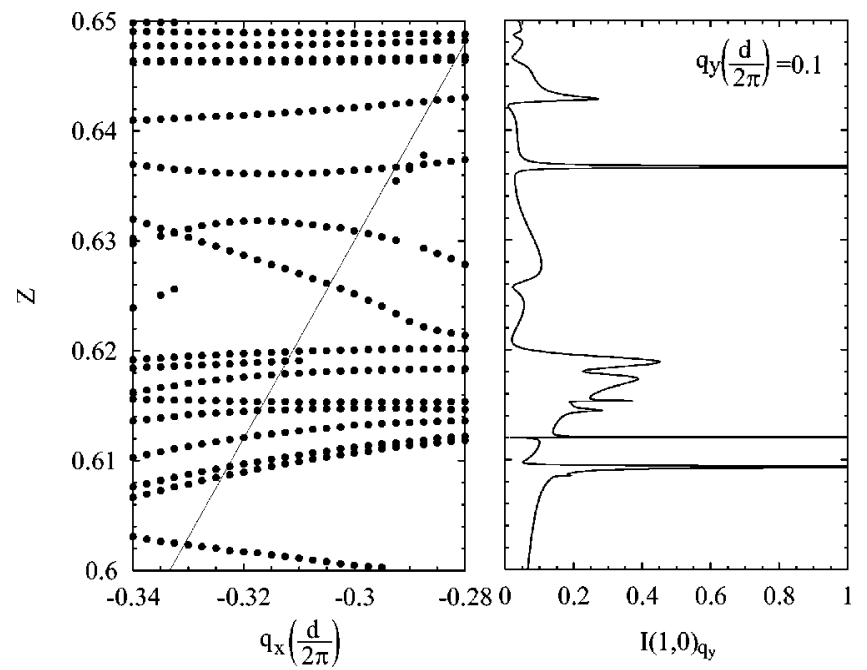

FIG. 5. Band structure and SPR spectrum for a nonzero $q_{y}$. The results for $N=4, \beta=0.9$ and $q_{y}=0.1(2 \pi / d)$ are given. The left panel shows the band structure for $\mathbf{q}=\left[q_{x}, 0.1(2 \pi / d)\right]$. The straight line shows the $h_{10}$ line, whose points of intersection with the dispersion curves leave their traces without exception in the SPR intensity curve shown in the right panel.

$=\left(k_{x}, q_{y}\right)$, with $k_{x}$ given by Eq. (3), is not on the symmetry axis of the 2D Brillouin zone, the excited photonic bands are no longer classified into $p$ - or $s$-active bands. Figure 5 shows the spectra of $I(1,0)_{q_{y}}$ with $q_{y}=0.1(2 \pi / d)$, together with the corresponding band structure. As expected, a resonant peak appears in $I(1,0)_{q_{y}}$ at any crossing point of the band structure. For a typical frequency $Z=0.62$, the value $q_{y}$ $=0.1(2 \pi / d)$ corresponds to the direction of SP light tilted $\sim 10^{\circ}$ away from the $x-z$ plane.

We have checked that the positions of the resonant structures in the SPR spectrum are a faithful replica of the band structure. In this sense, the SPR could be a useful tool to determine the band structure of photonic crystals. To obtain the $q_{x}$ dependence of the band dispersion, we have only to vary the electron velocity to change the tangent of the $h_{10}$ line. If we tilt the direction of the observation in the $y$ direction, we can even get the $q_{y}$ dependence of the band energies.

\section{Effect of the change of the direction of the electron beam}

Finally we consider the case of an electron beam traveling in a direction not parallel to the symmetry axis. Figure 6(a) shows the direction of the electron beam in the $q_{x}-q_{y}$ plane that has an angle $\phi_{\mathrm{el}}$ off the $x$ axis. In the rotated coordinate system $x^{\prime}$ and $y^{\prime}$ with the $x^{\prime}$ axis in the beam direction, the evanescent wave emitted by the electron is expressed in the same way as that in Eq. (7) with $k_{x}^{\prime}$ fixed at $k_{x}^{\prime}=\omega / v$ and has a form of a superposition over the momentum component $q_{y}^{\prime}$. For the light of frequency $\omega$, the point $\Omega_{\mathrm{i}}\left(k_{x}^{\prime}, q_{y}^{\prime}\right)$ of the incident evanescent wave is marked in Fig. 6(a), with the dashed circle of radius $\omega / c$ showing the locus of the light cone with the plane at the frequency height $\omega$. The incident field is then umklapp scattered by the photonic crystal. The

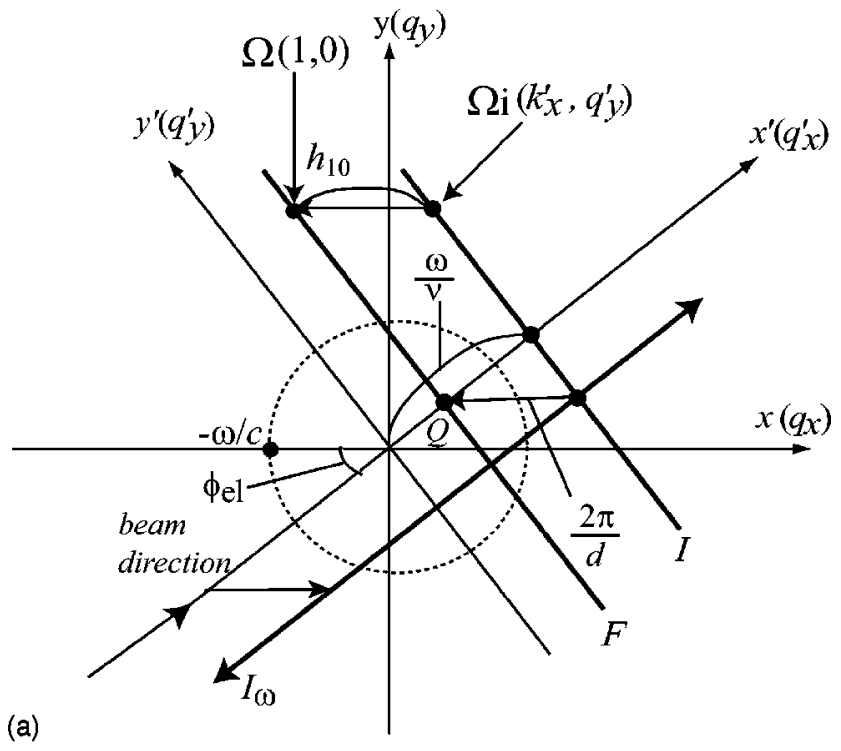

(b)

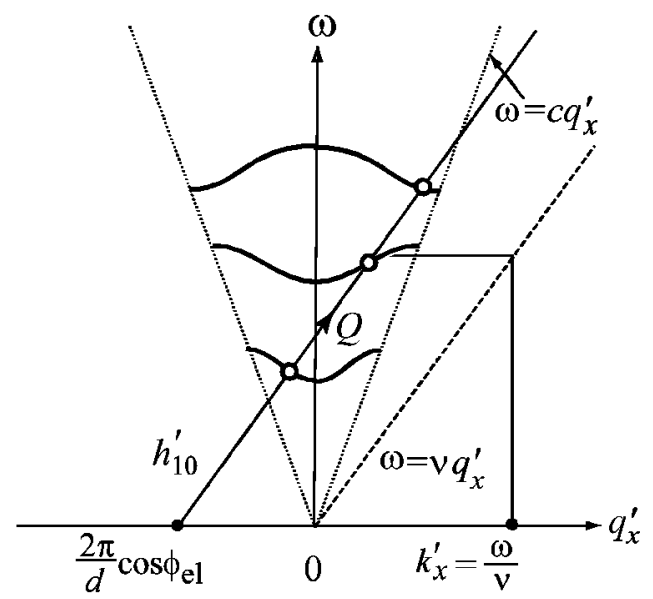

FIG. 6. Diagram showing the procedure of obtaining an SPR spectrum from an electron running off the $x$ axis. In (a), the axis $q_{x}^{\prime}$ represents the direction of the electron, which has an angle $\phi_{\mathrm{el}}$ from the $x$ direction. Line $I_{\omega}$ is the trajectory in the $\mathbf{q}$ space of the initial wave vector of the evanescent wave, when the frequency $\omega$ is varied for the SPR, which is observed in the plane spanned by the $x^{\prime}$ and $z$ axes. For the definitions of the other notations, see the text. Panel (b) shows a diagram similar to Fig. 2 in the $q_{x}^{\prime}-\omega$ plane, $q_{x}^{\prime}$ axis being defined in (a). Several model dispersion curves are given.

momentum of the outgoing light of frequency $\omega$ is shown for the case of $(1,0)$ umklapp shift at point $\Omega(1,0)$. In accordance with the arbitrariness of $q_{y}^{\prime}$ on the line $I$ (initial), the $h_{10}$ SPR spectrum will appear along the straight line $F$ (final) bounded by the light cone shown by the dashed circle. With change in $\omega$, the line $F$ shifts in accordance with the parallel movement of line $I$.

If we observe the emitted radiation above the beam trajectory, the 2D wave vector $\left[\left(\mathbf{k}_{s}\right)_{x},\left(\mathbf{k}_{s}\right)_{y}\right]$ of the detected light should lie on the $x^{\prime}$ axis. In this setting, therefore, sweeping $\omega$ of the observed light corresponds to varying the initial wave vector along the line denoted by $I_{\omega}$, which intersects the $q_{y}^{\prime}$ axis at $q_{y}^{\prime}=-(2 \pi / d) \sin \phi_{\mathrm{el}}$. Thus, the $h_{10}$ SPR spectrum provides information on the band structure for 
$\mathbf{q}$ on the $I_{\omega}$ line. Due to the periodicity in the $\mathbf{q}$ space, this $h_{10}$ line carries information along the $q_{x}^{\prime}$ axis.

Figure 6(b) shows the line $\omega=v q_{x}^{\prime}$ in the $q_{x}^{\prime}-\omega$ plane and the movement of point $Q$ shown in Fig. 6(a) along the straight line denoted by $h_{10}^{\prime}$. Together with these lines, we illustrate in Fig. 6(b) several model dispersion curves of the photonic bands for $\mathbf{q}$ on the $q_{x}^{\prime}$ axis. The SPR spectrum now consists of sharp resonant peaks arising at the crossing points of the $h_{10}^{\prime}$ line with the band dispersion curves. A clear-cut selection rule for the polarization as shown in Fig. 3(c) is not observed in the case of a tilted beam direction, because the $q_{x}^{\prime}$ axis is now in a general direction and the excited photonic bands are at general points of the 2D Brillouin zone. From this consideration, we may concluded that scanning $\phi_{\mathrm{el}}$ and studying the SPR spectrum will provide information on the photonic bands over the 2D Brillouin zone.

\section{EMISSION CROSS SECTION OF THE SPR}

\section{A. Expression of emission cross section of the SPR}

In Sec. III, we examined $I\left(n_{x}, n_{y}\right)_{q_{y}}$ in arbitrary units. Here we are interested in the actual magnitude of the photon yield as a function of the direction of observation in order to establish a comparison with a diffraction grating. We consider in this section that the electron beam moves along the $x$ axis.

Starting with the expression of the $z$ component of the Poynting vector of the emitted light

$$
\begin{aligned}
W= & \sum_{\mathbf{h}} W(\mathbf{h})=\sum_{\mathbf{h}} \frac{d}{\pi^{2}} \int_{0}^{\infty} \int_{-\infty}^{\infty} d \omega d q_{y} \frac{1}{2} \operatorname{Re}\{\hat{z} \cdot[\boldsymbol{E}(\mathbf{h}) \\
& \left.\left.\times \boldsymbol{H}(\mathbf{h})^{*}\right]\right\},
\end{aligned}
$$

we divide the Poynting vector into the contributions from the $2 \mathrm{D}$ reciprocal lattice vectors $\mathbf{h}$ of the umklapp scattering by expressing the emitted electric field as

$$
\boldsymbol{E}_{s}(\mathbf{r}, t)=\sum_{\mathbf{h}} \int_{-\infty}^{\infty} \frac{d \omega}{2 \pi} \int_{-\infty}^{\infty} \frac{d q_{y}}{2 \pi} \boldsymbol{E}(\mathbf{h}) \exp \left\{i\left[\mathbf{k}_{s}(\mathbf{h}) \cdot \mathbf{r}-\omega t\right]\right\},
$$

which is the Fourier transform of Eq. (14). The magnetic field $\boldsymbol{H}(\mathbf{h})$ is defined analogously. Expression (26) of $W$ is obtained by integrating the Poynting vector over one period $d$ in the $x$ direction. The radiated energy $W$ should be equal to the kinetic energy of the electron lost by the photon emission. It can indeed be shown that $W$ is just equal to the work required to keep the velocity of electron constant in opposition to the force due to $\boldsymbol{E}_{s}(\mathbf{r}, t)$ seen by the electron, ${ }^{16}$ i.e.,

$$
W=\int_{t_{0}}^{t_{0}+d / v} d t e v\left[\boldsymbol{E}_{s}(v t, t)\right]_{x},
$$

the integral being over the time required for the electron to traverse the distance $d$.

We introduce the polar angles $\theta(\mathbf{h})$ and $\phi(\mathbf{h})$ for the emission direction, which are defined in Fig. 7 relative to the beam direction,

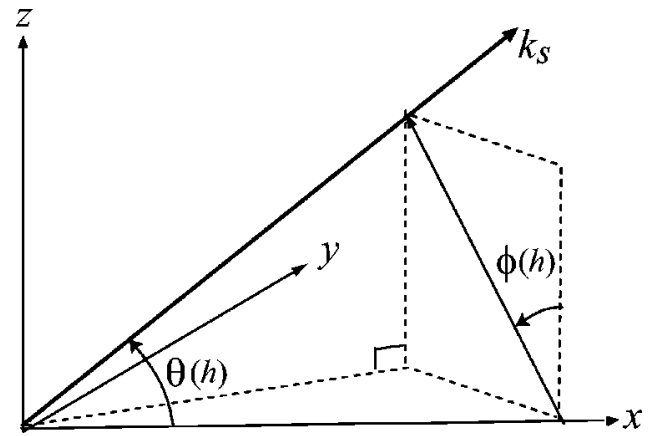

FIG. 7. Angles $\theta(\mathbf{h})$ and $\phi(\mathbf{h})$ defined by Eq. (29). $\mathbf{k}_{s}$ is the wave vector of the emitted photon defined by Eq. (12).

$$
\left[\mathbf{k}_{s}(\mathbf{h})\right]_{x} \equiv \frac{\omega}{v}+h_{x}=\frac{\omega}{c} \cos \theta(\mathbf{h}),
$$

$$
\left[\mathbf{k}_{s}(\mathbf{h})\right]_{y} \equiv q_{y}^{\prime}=q_{y}+h_{y}=\frac{\omega}{c} \sin \theta(\mathbf{h}) \sin \phi(\mathbf{h}),
$$

$$
\left[\mathbf{k}_{s}(\mathbf{h})\right]_{z}=\frac{\omega}{c} \sin \theta(\mathbf{h}) \cos \phi(\mathbf{h}) .
$$

For a fixed $\mathbf{h}$, the direction of emission specified by $[\theta(\mathbf{h}), \phi(\mathbf{h})]$ changes continuously as $\omega$ and $q_{y}$ vary. Accordingly, we can define the emission probability per unit solid angle of observation. From the first and second of Eq. (29), the Jacobian for the $\omega$ and $q_{y}$ integrals of Eq. (26) is obtained as

$$
J(\mathbf{h})=\left|\begin{array}{cc}
\frac{\partial q_{y}^{\prime}}{\partial \theta(\mathbf{h})} & \frac{\partial \omega}{\partial \theta(\mathbf{h})} \\
\frac{\partial q_{y}^{\prime}}{\partial \phi(\mathbf{h})} & \frac{\partial \omega}{\partial \phi(\mathbf{h})}
\end{array}\right|=\frac{(2 \pi)^{3} c}{\lambda^{3} h_{x}} \sin ^{3} \theta(\mathbf{h}) \cos \phi(\mathbf{h}),
$$

where $\lambda=(2 \pi c / \omega)$ is the wavelength of the observed photon. From Eqs. (26) and (30), it holds that

$$
\begin{aligned}
W(\mathbf{h})= & \int_{0}^{\pi} \sin \theta(\mathbf{h}) d \theta(\mathbf{h}) \\
& \times \int_{0}^{2 \pi} d \phi(\mathbf{h}) \Xi(\mathbf{h})|\Lambda(\mathbf{h})|^{2} e^{-2|\Gamma|(D+a),}
\end{aligned}
$$

where

$$
\Xi(\mathbf{h})=\pi \frac{e^{2}}{\epsilon_{0}}\left(\frac{d}{\lambda^{3} h_{x}}\right) \sin ^{2} \theta(\mathbf{h}) \cos ^{2} \phi(\mathbf{h})
$$

and $\left(\varepsilon_{0} \mu_{0}=1 / c^{2}\right)$

$$
\begin{aligned}
|\Lambda(\mathbf{h})|^{2}= & \frac{8 e^{2|\Gamma|(D+a)}}{\mu_{0} c e^{2} \sin \theta(\mathbf{h}) \cos \phi(\mathbf{h})} \\
& \times \frac{1}{2} \operatorname{Re}\left\{\hat{z} \cdot\left[\boldsymbol{E}(\mathbf{h}) \times \boldsymbol{H}(\mathbf{h})^{*}\right]\right\} .
\end{aligned}
$$


Since $(1 / 2) \operatorname{Re}[\cdots]$ involves in itself the decaying factor $e^{-2|\Gamma|(D+a)}$, the quantity $|\Lambda(\mathbf{h})|^{2}$ defined by Eq. (33) is free from the $D$ dependence and describes the intrinsic properties of the light conversion through the photonic band excitation. This quantity was referred to as the radiation factor in Refs. 13,14 .

The power emitted into a unit solid angle while the electron traversing one period $d$ is then given by

$$
\frac{d W(\mathbf{h})}{d \Omega}=\Xi(\mathbf{h})|\Lambda(\mathbf{h})|^{2} e^{-2|\Gamma|(D+a)},
$$

which we shall call simply the SPR cross section. The quantity $\Xi$ (h) defined by Eq. (32) has the dimension of energy and varies with $\omega$ slowly and smoothly through the factor $\lambda^{-3}$. The main part of the cross section related to the photonic band structure is thus the radiation factor $|\Lambda(\mathbf{h})|^{2}$. Indeed, the quantity $I\left(n_{x}, n_{y}\right)_{q_{y}}$, which we called the SPR intensity in Sec. III, is found to be

$$
I\left(n_{x}, n_{y}\right)_{q_{y}}=\left|\Lambda\left(n_{x}, n_{y}\right)\right|^{2} e^{-2|\Gamma|(D+a)},
$$

where we employ the index $\left(n_{x}, n_{y}\right)$ for $\mathbf{h}$ of $\Lambda(\mathbf{h})$ and the value of $q_{y}$ on the left-hand side is fixed by the second relation of Eq. (29) as a function of $\mathbf{h}$ and $[\theta(\mathbf{h}), \phi(\mathbf{h})]$. As a result the cross section and intensity in our definition are related as

$$
\frac{d W(\mathbf{h})}{d \Omega}=\Xi(\mathbf{h}) I\left(n_{x}, n_{y}\right)_{q_{y}} .
$$

\section{B. Comparison between $I$ and $d W / d \Omega$}

The case of $\phi(\mathbf{h})=0$ corresponds to the vertical emission of $\left(\mathbf{k}_{s}\right)_{y}=0$ considered in Secs. III A and III B. For the case of $\mathbf{h}=(2 \pi / d)(1,0)$ and $q_{y}=0$, we compare the cross section $d W(\mathbf{h}) / d \Omega$ and the intensity $I(1,0)_{0}$. We consider the photonic crystal with $N=1$, for simplicity. Figure 8 (a) shows $I(1,0)_{0}$ in arbitrary units as in Sec. III and Fig. 8(b) shows the cross section $d W(\mathbf{h}) / d \Omega$ given by Eq. (34). As in Sec. III, $a / d=0.42, D / d=0.5, \beta=0.9$, and $\varepsilon=3.2^{2}$. We see at once the similarity between them. From the relation given by Eq. (36), the difference in the $Z$ dependence should come solely from the factor $Z^{3}\left(\sim \lambda^{-3}\right)$ involved in the factor $\Xi$ (h) defined by Eq. (32). The reason why the cross section $d W(\mathbf{h}) / d \Omega$ still decreases even in the presence of the increasing factor $Z^{3}$ is that the enhanced damping of the evanescent wave with increasing $Z$ dominates and overshoots the increase. Indeed, in the case of a smaller $D$, where the damping is less dominant, the effect of the factor $Z^{3}$ becomes more appreciable in the cross section. Figure $8(\mathrm{c})$, which shows the extreme case of $D=0$, illustrates this situation. Note, however, that the decrease due to the exponential damping still works even in the limit $D=0$, since it depends not on $e^{-2|\Gamma| D}$, but on $e^{-2|\Gamma|(D+a)}$. This is why $d W / d \Omega$ does not increase in Fig. 8(c) in spite of the $Z^{3}$ increase.

The conclusion is thus that apart from the absolute magnitudes of the photon yield and the slowly varying frequency dependence due to $Z^{3}$, we can safely employ the quantity
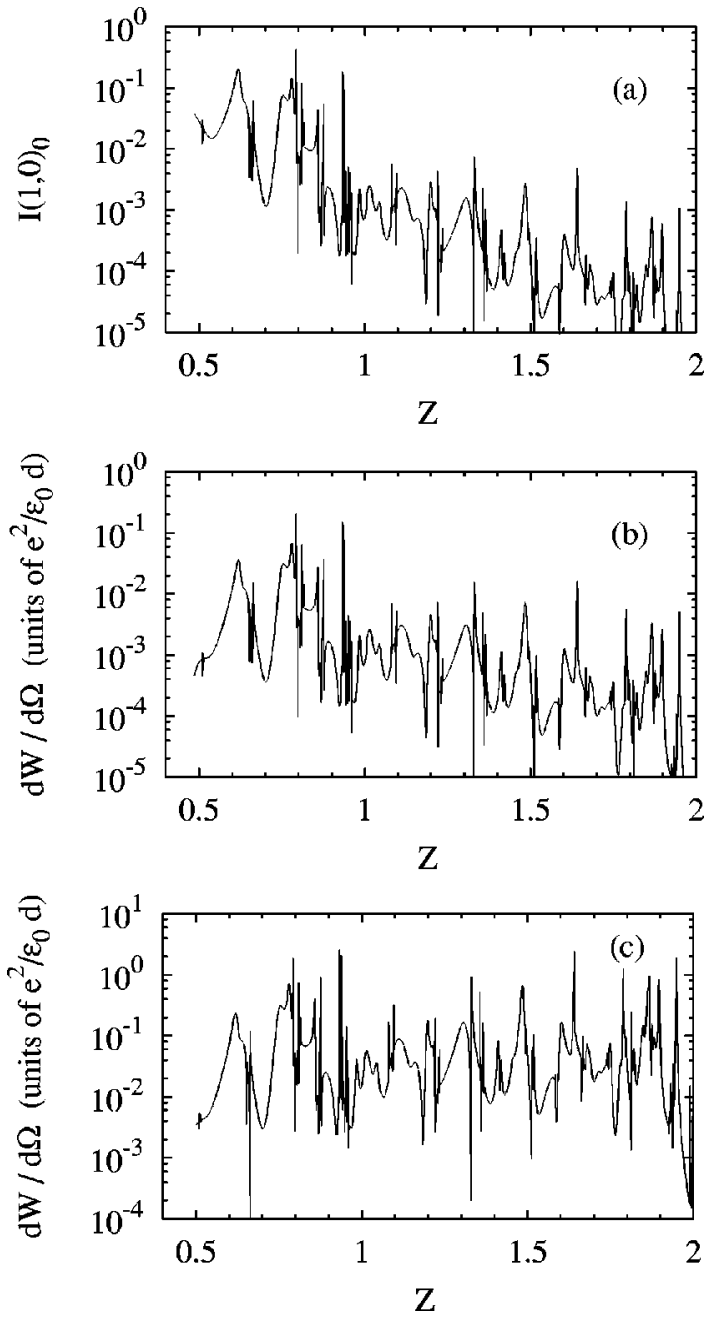

FIG. 8. Comparison between the SPR intensity and cross section for the photonic crystal of $N=1$ and for $\beta=0.9$. The panel (a) shows $I(1,0)_{0}$ and (b) shows $d W / d \Omega$ for $\mathbf{h}=(2 \pi / d)(1,0)$ as observed in the $x-z$ plane. The results shown in (a) and (b) are for $D / d=0.5$. In (c), $d W / d \Omega$ of $\mathbf{h}=(2 \pi / d)(1,0)$ is given for the limiting case of $D / d=0$ to see how the factor $Z^{3}$ involved in the cross section affects the $Z$ dependence.

$I\left(n_{x}, n_{y}\right)_{q_{y}}$ to examine the characteristic features of the SPR cross section from a photonic crystal. Our previous investigations, ${ }^{8}$ all made on $I\left(n_{x}, n_{y}\right)_{q_{y}}$, can thus be taken as indicating the essence of the SPR spectra from photonic crystals.

\section{Comparison between a photonic crystal and diffraction grating}

To see the characteristic features of photonic crystals compared with those of diffraction gratings, let us take as an example for the latter a perfectly conducting grating with rectangular groove profile. This type of the grating has often been considered in both theoretical and experimental works. To make the geometry of the two systems as similar as possible, we examine a photonic crystal of $N=1$ with $a / d$ $=0.25$ and a grating with groove width and height relative to 


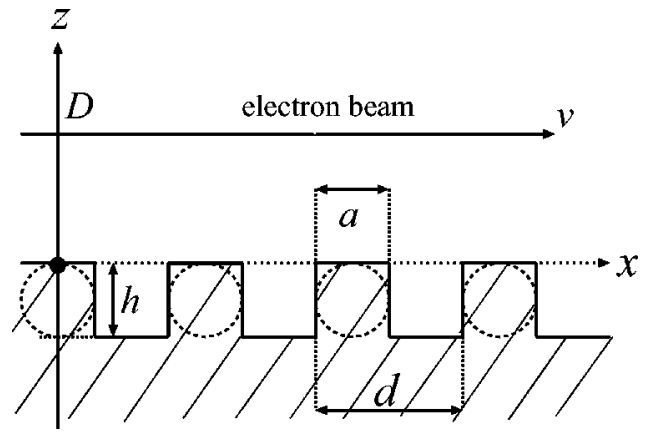

FIG. 9. Diffraction grating with a rectangular groove profile. A vertical figure of the photonic crystal of a free-standing 2D array of dielectric spheres is superimposed. The emission efficiencies of the two systems are compared in Figs. 10 and 11.

the grating period $d$ both equal to 0.5 . A vertical view of the two systems, when superimposed, is given in Fig. 9. The distance $D$ of the beam trajectory from the grating is measured from the top surface of the grating.

For the emission cross section of the $h_{10}$ SPR band, Fig. 10 compares the two systems for $\beta=0.9$ and $D=0.5 d$, in a logarithmic scale. The solid curve is for the photonic crystal and the dashed curve is for the grating. We can see a marked difference between the two curves both in magnitude and line shape. Numerous resonant peaks in the photonic crystal arise due to the photonic band excitations. It is notable that the heights of the sharp peaks are larger than those of the grating, generally by about one order of magnitude. Fine structures arise in the curves of the grating, too. In Fig. 10 we find the sharp dips and kinks appear at $Z$ $\simeq 0.9,1.4,1.9, \ldots$, together with broad peaks located between them. These are typical features observed experimentally in SPR spectra of gratings. ${ }^{11}$ It should, however, be noted that the peaks present in the grating case are too broad

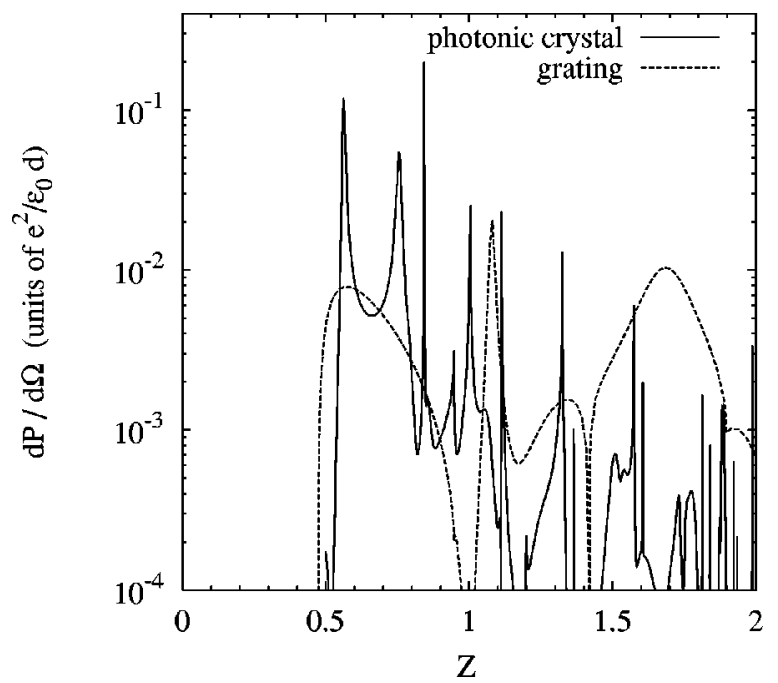

FIG. 10. Comparison of the cross sections of SPR between a photonic crystal and a perfectly conducting grating on a logarithmic scale. The results for $N=1, D / d=0.5$, and $q_{y}=n_{y}=0$ (i.e., for the $h_{10}$ SPR band) are given. The solid and dashed lines represent the spectra of the photonic crystal and of the grating, respectively. to compare their FWHM with those of the photonic-band excitation. Because of this, the peak heights of the grating SPR are much lower than those of the photonic crystal. The fine structures positioned at those values of $Z$ noted above appear when the $h_{10}$ line intersects the equally spaced $c$ lines associated with various umklapp shifts of integer multiples of $2 \pi / d$. Indeed, in our case of $\beta=0.9$, the crosses of the $h_{10}$ line with them are calculated to be $Z$ $=0.95,1.42,1.89, \ldots$, in very good agreement with the positions of the fine structures of Fig. 10. This shows that the band structure of the perfectly conducting grating is obtained by folding the light lines to the first Brillouin zone and that the quality factors of the excited bands are much smaller than the photonic bands of the arrayed dielectric spheres. This is another way of saying that the spectral difference is caused by the difference of the degree of the electromagnetic confinement.

Although we can obtain almost monochromatic and enhanced emission lines by using the excitation of the photonic bands, it should be pointed out that the integrated value of the spectral intensity of the photonic crystal does not look very different from that of the grating.

In Fig. 10, it is notable that the decay of the intensity with increase of $Z$ is different in the two cases. This is because the dependence on $|\Gamma|$ is through $e^{-|\Gamma|(D+a)}$ in the photonic crystal, while it is through $e^{-|\Gamma| D}$ in the grating. In the case of $D=0.5 d$ examined in Fig. 10, therefore, the decay of the grating is less appreciable. Indeed, for a larger value of $Z$ where $|\Gamma|$ becomes larger or a larger $D$ where the exponential decay is more appreciable than the $Z^{3}$ factor, we can confirm that the exponential decay prevails in the grating case, too.

Considering that the perfectly-conducting grating has $100 \%$ reflectance while the photonic crystal has a leak in the direction opposite to the electron beam, the above comparison was rather unfair to the photonic crystal; we may well think that if the monolayer array of spheres is placed on a perfect mirror to reflect the leaked light completely back to the photonic crystal, the peak values of the SPR cross section would become larger than the free-standing system treated in Fig. 10.

So far we have considered the photonic crystal of $N=1$. As we found in our previous work, ${ }^{28}$ the quality factor generally improves with $N$. Therefore, the positive conclusion obtained here for the peak values becomes more and more conspicuous, when a thicker slab photonic crystal is considered. This feature is confirmed in Fig. 11, which shows the cross section for $\mathbf{h}=(2 \pi / d)(1,0)$ for the slab of $N=1,2$, and 4. We see a sharp narrowing of the emission peaks, while simultaneously the peak intensities increase drastically.

\section{CONCLUSION}

In this paper we have examined the characteristic features of the SPR spectrum when a photonic crystal is used in place of a diffraction grating. We have treated a slab of photonic crystal, made out of stacking 2D layers of arrayed dielectric spheres. The procedure for obtaining the SPR spectra amounts in its essence to calculation of the diffracted ampli- 


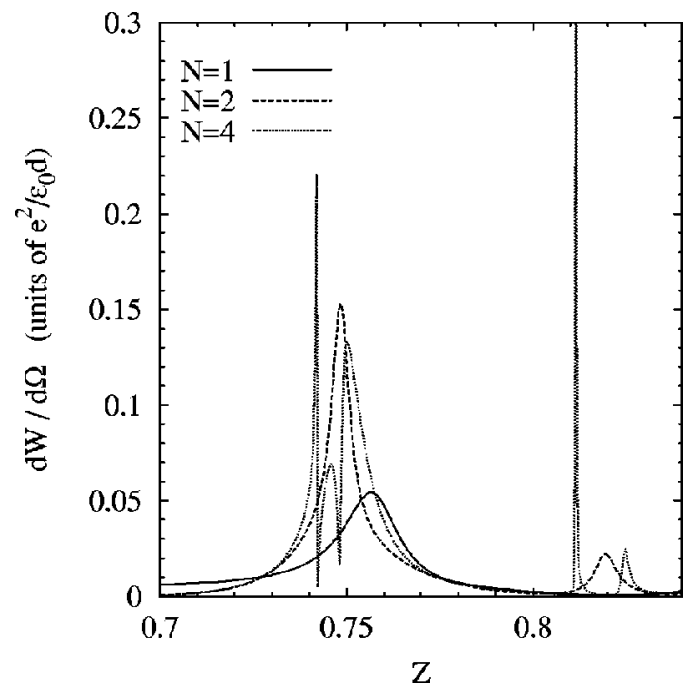

FIG. 11. $N$ dependence of the SPR cross section for a photonic crystal. The general tendency of a larger peak number and higher peak values with increase in number $N$ can be seen.

tudes of the incident evanescent field originally emitted by a traveling charge. We have treated this diffraction process with the multiple scattering of the light by the photonic crystal being taken into account exactly.

Features related to the selection rules characterizing the SPR spectra from the photonic crystal have been demonstrated. By varying the direction of observation of the emitted light, it was found that we can trace over the 2D Brillouin zone not only the dispersion surface but also the symmetry properties of a photonic band. It was also found that sweeping the direction of the electron beam relative to the symme- try axes of photonic crystals is equivalent to scanning the $2 \mathrm{D}$ Brillouin zone, as far as the 2D wave vectors of the excited photonic bands are concerned. Through changes in the positions and the widths of the resonant peaks, the SPR spectrum may serve as a good probe into a photonic band structure.

The SPR spectra are full of fine structures due to the excitations of photonic bands, while those of the grating have only the fine structures attributable to Wood anomalies, which takes place when a folded light-cone edge is excited by the periodic light scattering. In addition to the number of the fine structures, the sharpness of the resonance is quite contrasting, giving a marked difference in the peak values of the photon emission cross sections. We have made a quantitative comparison between a photonic crystal of arrayed dielectric spheres and a perfectly conducting grating of a rectangular groove profile. Compared to the emission lines of the diffraction grating, we can say that a photonic crystal gives rise to almost monochromatic emission lines in the SPR spectrum.

We have given a numerical calculation for a photonic crystal made out of arrayed spheres. Considering that the measured transmittance of the $N=1$ photonic crystal of arrayed $\mathrm{Si}_{3} \mathrm{~N}_{4}$ spheres of $\mathrm{mm}$ range agrees remarkably well with the calculated prediction, ${ }^{30}$ we expect the comparison in SPR spectrum to also be favorable.

\section{ACKNOWLEDGMENTS}

The authors acknowledge the financial support provided by the Grant-in-Aid for Specific Scientific Area from the Ministry of Education, Sports, Culture, Science and Technology of Japan. The authors also thank the same ministry for another grant for "Promotion of Science and Technology".
${ }^{1}$ S.J. Smith and E.M. Purcell, Phys. Rev. 92, 1069 (1953).

${ }^{2}$ K. Mizuno, S. Ono, and O. Shimoe, Nature (London) 253, 184 (1975).

${ }^{3}$ R.H. Pantell, Nucl. Instrum. Methods Phys. Res. A 393, 1 (1997); M.Q. Lu, Y. Cheng, Z.Z. Xu, and S. Wang, Phys. Plasmas 5, 825 (1998).

${ }^{4}$ J.M. Wachtel, J. Appl. Phys. 50, 49 (1979); R.P. Leavitt and D.E. Wortman, ibid. 54, 2219 (1983); J. Soln and R.P. Leavitt, ibid. 56, 29 (1984).

${ }^{5}$ T. Shiozawa and M. Sata, Appl. Phys. Lett. 66, 124 (1995); I.V. Ivanchenko, Microwave Opt. Technol. Lett. 20, 21 (1999); A.W. Cross, I.V. Konoplev, K. Ronald, A.D.R. Phelps, W. He, C.G. Whyte, N.S. Ginzburg, N.Yu. Peskov, and A.S. Sergeev, Appl. Phys. Lett. 80, 1517 (2002), and the references therein.

${ }^{6}$ E. Yablonovitch, Phys. Rev. Lett. 58, 2059 (1987).

${ }^{7}$ See, for example, J. Lightwave Technol. 17(11) (1999), which is one of the most recent feature issues of photonic crystals, and the references therein.

${ }^{8}$ K. Ohtaka and S. Yamaguti, Opt. Quantum Electron. 34, 235 (2002); Opt. Spectrosc. 91, 477 (2001).

${ }^{9}$ J.H. Brownell, J. Walsh, H.G. Kirk, R.C. Fernow, and S.H. Robertson, Nucl. Instrum. Methods Phys. Res. A 393, 323 (1997);
J.H. Brownell, J. Walsh, and G. Doucas, Phys. Rev. E 57, 1075 (1998).

${ }^{10}$ A.P. Potylitsyn, Nucl. Instrum. Methods Phys. Res. B 145, 60 (1998); C.H. Lee, J.I. Lee, U.H. Cho, and Y.K. Cho, Microwave Opt. Technol. Lett. 19, 292 (1998); C.S. Liu and V.K. Tripathi, IEEE J. Quantum Electron. 35, 1386 (1999); J.E. Walsh, Nucl. Instrum. Methods Phys. Res. A 445, 214 (2000); H. Ishizuka, Y. Kawamura, K. Yokoo, H. Shimawaki, and A. Hosono, ibid. 445, 276 (2000).

${ }^{11}$ For the SPR as a coherent light source, see, e.g., Y. Shibata, S. Hasebe, K. Ishi, S. Ono, M. Ikezawa, T. Nakazato, M. Oyamada, S. Urasawa, T. Takahashi, T. Matsuyama, K. Kobayashi, and Y. Fujita, Phys. Rev. E 57, 1061 (1998), and the references therein. For the radiation from SEM involving induced emission from a grating, see, J. Urata, M. Goldstein, M.F. Kimmitt, A. Naumov, C. Platt, and J.E. Walsh,, Phys. Rev. Lett. 80, 516 (1998).

${ }^{12}$ For recent works and references, see, e.g., Y-H. Liu and T.C. Marshall, Phys. Rev. E 56, 2161 (1997); K. Kamada, K. Nawashiro, F. Tamagawa, H. Igarashi, S. Kizu, C-Y. Lee, S. Kawasaki, R. Ando, and M. Masuzaki, Int. J. Infrared Millim. Waves 19, 1317 (1998); F. Lewen, R. Gendriesch, I. Pak, D.G. Paveliev, M. Hepp, R. Schieder, and G. Winnewisser, Rev. Sci. Instrum. 69, 32 (1998). 
${ }^{13}$ O. Haeberlé, P. Rullhusen, J.-M. Salomé, and N. Maene, Phys. Rev. E 49, 3340 (1994).

${ }^{14}$ O. Haeberlé, P. Rullhusen, J.-M. Salomé, and N. Maene, Phys. Rev. E 55, 4675 (1997).

${ }^{15}$ F.J. Garcia de Abajo, Phys. Rev. Lett. 82, 2776 (1999); Phys. Rev. E 61, 5743 (2000)

${ }^{16}$ P.M. van den Berg, J. Opt. Soc. Am. 63, 689 (1973); 63, 1588 (1973); 64, 325 (1974).

${ }^{17}$ R.W. Wood, Philos. Mag. 4, 396 (1902).

${ }^{18}$ J.W.S. Rayleigh, Philos. Mag. 14, 60 (1907).

${ }^{19}$ L. D. Landau and E. M. Lifshitz, Quantum Mechanics, 2nd ed. (Pergamon, London, 1965), p. 565.

${ }^{20}$ R. Petit, Electromagnetic Theory of Gratings (Springer-Verlag, Berlin, 1980).

${ }^{21}$ K. Ohtaka, Phys. Rev. B 19, 5057 (1979).

${ }^{22}$ K. Ohtaka, J. Phys. C 13, 667 (1980).

${ }^{23}$ N. Stefanou, V. Karathanos, and A. Modinos, J. Phys.: Condens. Matter 4, 7389 (1992); V. Yannopapas, A. Modinos, and N. Ste- fanou, Phys. Rev. B 60, 5359 (1999); A. Modinos, N. Stefanou, and V. Yannopapas, Opt. Express 8, 197 (2001).

${ }^{24}$ K. Ohtaka and Y. Tanabe, J. Phys. Soc. Jpn. 65, 2276 (1996).

${ }^{25}$ For a recent work related to WGM, see, e.g., T. Mukaiyama, K. Takeda, H. Miyazaki, Y. Jimba, and M. Kuwata-Gonokami, Phys. Rev. Lett. 82, 4623 (1999), and the references therein.

${ }^{26}$ K. Ohtaka and Y. Tanabe, J. Phys. Soc. Jpn. 65, 2670 (1996).

${ }^{27}$ K. Ohtaka, Y. Suda, S. Nagano, T. Ueta, A. Imada, T. Koda, J.S. Bae, K. Mizuno, S. Yano, and Y. Segawa, Phys. Rev. B 61, 5267 (2000).

${ }^{28}$ K. Ohtaka, J. Lightwave Technol. 17, 2161 (1999).

${ }^{29} \mathrm{We}$ are planning the experiment using a photonic crystal of $\mathrm{Si}_{3} \mathrm{~N}_{4}$ spheres of millimeter size of dielectric constant $\sim 3.0^{2}$. If we use the normalized frequency units $Z$, the results of this paper apply without modification.

${ }^{30}$ T. Kondo, M. Hangyo, S. Yamaguti, S. Yano, Y. Segawa, and K. Ohtaka, Phys. Rev. B 66, 033111 (2002). 